\title{
Conservation of energy for the Euler-Korteweg equations
}

\author{
Tomasz Dębiec ${ }^{1} \cdot$ Piotr Gwiazda $^{2}$ - Agnieszka Świerczewska-Gwiazda ${ }^{1}$ • \\ Athanasios Tzavaras ${ }^{3}$
}

Received: 23 December 2017 / Accepted: 7 September 2018 / Published online: 29 September 2018

(c) The Author(s) 2018

\begin{abstract}
In this article we study the principle of energy conservation for the Euler-Korteweg system. We formulate an Onsager-type sufficient regularity condition for weak solutions of the EulerKorteweg system to conserve the total energy. The result applies to the system of Quantum Hydrodynamics.
\end{abstract}

Mathematics Subject Classification 76D45 · 35G50

\section{Introduction}

It is known since the works of Scheffer [28] and Shnirelmann [29] that weak solutions of the incompressible Euler equations exhibit behaviour very different to that of classical solutions. These "wild solutions", as they are called since the seminal works of DeLellis and Székelyhidi $[10,11]$, are often highly unphysical—for instance there is a lack of uniqueness and the principle of conservation of energy can be violated.

Dissipative solutions of incompressible Euler have been extensively studied in relation to the seminal Onsager conjecture [27]. It states that there is a threshold regularity, namely $\frac{1}{3}$-Hölder continuity, above which kinetic energy must be conserved, and below which

Communicated by C. De Lellis.

\footnotetext{
Agnieszka Świerczewska-Gwiazda

aswiercz@mimuw.edu.pl

Tomasz Dębiec

t.debiec@mimuw.edu.pl

Piotr Gwiazda

pgwiazda@mimuw.edu.pl

Athanasios Tzavaras

athanasios.tzavaras@kaust.edu.sa

1 Institute of Applied Mathematics and Mechanics, University of Warsaw, Banacha 2, 02-097 Warsaw, Poland

2 Institute of Mathematics, Polish Academy of Sciences, Śniadeckich 8, 00-656 Warsaw, Poland

3 Computer, Electrical, Mathematical Sciences and Engineering Division, King Abdullah University of Science and Technology (KAUST), Thuwal, Saudi Arabia
} 
anomalous dissipation might occur. This conjecture has been recently fully resolved, with non-conservative solutions of class $\mathscr{C}\left([0, T] ; \mathscr{C}^{\frac{1}{3}-}\left(\mathbb{T}^{3}\right)\right)$ constructed by Isett [23]. See also $[5,24]$ for further developments on the subject.

The positive direction of Onsager's conjecture has been settled already in the 1990's by Constantin et al. [9] (after a partial result of Eyink [17]). The method of mollification and estimation of commutator errors was employed to prove that, if a weak solution $u$ of the incompressible Euler system belongs to $L^{3}\left([0, T], B_{3}^{\alpha, \infty}\left(\mathbb{T}^{3}\right)\right) \cap \mathscr{C}\left([0, T], L^{2}\left(\mathbb{T}^{3}\right)\right)$ with $\alpha>\frac{1}{3}$, then the energy $\|u\|_{L^{2}\left(\mathbb{T}^{3}\right)}$ is conserved in time. The method of proof as well as the observation that Besov spaces provide a suitable environment for this kind of problem were later used by several authors in the context of other systems of fluid dynamics: like inhomogeneous incompressible Euler and compressible Euler [18], incompressible and compressible Navier-Stokes (resp. [14,15,26,31]), incompressible magnetohydrodynamics [8,25], and general systems of first order conservation laws [22]. Onsager's conjecture was recently studied for incompressible Euler equations in bounded domains, cf. [3]. An overview of these results can be found in [12].

In the present paper we adapt the strategy of Constantin et al. [9] and Feireisl et al. [18] to obtain an Onsager-type sufficient condition on the regularity of weak solutions to the Euler-Korteweg equations so that they conserve the total energy. We consider the isothermal Euler-Korteweg system in the from

$$
\begin{aligned}
\partial_{t}(\rho u)+\operatorname{div}(\rho u \otimes u) & =-\rho \nabla\left(h^{\prime}(\rho)+\frac{\kappa^{\prime}(\rho)}{2}|\nabla \rho|^{2}-\operatorname{div}(\kappa(\rho) \nabla \rho)\right), \\
\partial_{t} \rho+\operatorname{div}(\rho u) & =0,
\end{aligned}
$$

in the domain $(0, T) \times \mathbb{T}^{d}$ for some fixed time $T>0$, where $\mathbb{T}^{d}$ is the $d$-dimensional torus. Here $\rho \geq 0$ is the scalar density of a fluid, $u$ is its velocity, $h=h(\rho)$ is the energy density and $\kappa=\kappa(\rho)>0$ is the coefficient of capillarity. We place the assumption on the functions $h$ and $\kappa$ :

$$
h, \kappa \in \mathscr{C}^{3}(\mathscr{T})
$$

where, depending on the actual form of $h$ and $\kappa$, the set $\mathscr{T}$ can be chosen to be $[0, \infty)$ or $(0, \infty)$. For instance when $\kappa(\rho)=\frac{1}{\rho}$, as for the QHD system below, then $\mathscr{T}=(0, \infty)$ and we have to be away from vacuum.

While the analysis of the above system dates back to the 19th century, when the mathematical theory of phase interfaces and capillary effects was introduced, it still attracts much attention. A modern derivation of the system can be found in [16]. Concerning smooth solutions: in [6,7] local-in-time well-posedness and stability of special solutions are analysed, respectively. A relative energy identity is developed in [21], exploiting the variational structure of the system, and is used to show that solutions of (1.1) converge to smooth solutions of the compressible Euler system (before shock formation) in the vanishing capillarity limit $\kappa \rightarrow 0$, see [20].

The situation with weak solutions is much less understood. Most results concern the Quantum Hydrodynamics system, obtained from (1.1) when $\kappa(\rho)=\frac{\varepsilon_{0}^{2}}{4 \rho}$, with $\varepsilon_{0}$ denoting the Planck constant. This takes the form

$$
\begin{aligned}
\partial_{t} \rho+\operatorname{div}(\rho u) & =0, \\
\partial_{t}(\rho u)+\operatorname{div}(\rho u \otimes u)+\nabla p(\rho) & =\frac{\varepsilon_{0}^{2}}{2} \rho \nabla\left(\frac{\Delta \sqrt{\rho}}{\sqrt{\rho}}\right) .
\end{aligned}
$$


The interesting connection between QHD and the Schrödinger equation is used in [19] to provide conservative weak solutions for the special case of zero pressure, $p(\rho)=0$. Existence of weak solutions for a (relatively limited) class of pressure functions is provided in [1,2]. The existence of wild solutions is possible for (1.1), as pointed out in the recent work of Donatelli et al. [13], where the method of "convex integration" is adapted to show non-uniqueness in the class of dissipative global weak solutions.

The possibility of both conservative and dissipative solutions raises the issue of studying the Onsager conjecture for the Euler-Korteweg system (1.1). We use Besov spaces $B_{p}^{\alpha, \infty}(\Omega)$, with $1 \leq p<\infty, 0<\alpha<1$ (see Sect. 2.1 for the definition) and prove the following theorem:

Theorem 1.1 Suppose that (1.2) holds. Let $(\rho, u)$ be a solution of (1.1) in the sense of distributions. Assume

$$
u \in\left(B_{3}^{\alpha, \infty} \cap L^{\infty}\right)\left((0, T) \times \mathbb{T}^{d}\right), \quad \rho, \nabla \rho, \Delta \rho \in\left(B_{3}^{\beta, \infty} \cap L^{\infty}\right)\left((0, T) \times \mathbb{T}^{d}\right),
$$

where $1>\alpha \geq \beta>0$ such that $\min (2 \alpha+\beta, \alpha+2 \beta)>1$.

Then the energy is locally conserved, i.e.

$$
\begin{aligned}
\int_{0}^{T} \int_{\mathbb{T}^{d}}( & \left.\frac{1}{2} \rho|u|^{2}+h(\rho)+\frac{1}{2} \kappa(\rho)|\nabla \rho|^{2}\right) \partial_{t} \varphi d x d t \\
& +\int_{0}^{T} \int_{\mathbb{T}^{d}}\left(\rho u\left(\frac{1}{2}|u|^{2}+h^{\prime}(\rho)+\frac{1}{2} \kappa^{\prime}(\rho)|\nabla \rho|^{2}-\operatorname{div}(\kappa(\rho) \nabla \rho)\right)\right. \\
& +\kappa(\rho) \nabla \rho \operatorname{div}(\rho u)) \cdot \nabla \varphi d x d t=0
\end{aligned}
$$

holds for every $\varphi \in \mathscr{C}_{c}^{1}\left((0, T) \times \mathbb{T}^{d}\right)$.

Remark 1.2 (1) Notice that if $\alpha \geq \beta$, then $3 \alpha \geq \alpha+2 \beta>1$ and so we must have $\alpha>\frac{1}{3}$.

(2) If in addition we assume the following conditions on $u$ and $\rho$

$$
\begin{aligned}
& \lim _{|\xi|, \tau \rightarrow 0} \frac{1}{\tau} \int_{0}^{T} \frac{1}{|\xi|} \int_{\mathbb{T}^{d}}|u(t+\tau, x+\xi)-u(t, x)|^{3} d x d t=0, \\
& \lim _{|\xi|, \tau \rightarrow 0} \frac{1}{\tau} \int_{0}^{T} \frac{1}{|\xi|} \int_{\mathbb{T}^{d}}|\rho(t+\tau, x+\xi)-\rho(t, x)|^{3} d x d t=0,
\end{aligned}
$$

then, as pointed out by Shvydkoy [30], see also Duchon and Robert [15], one can allow for the case $\alpha=\beta=\frac{1}{3}$. For details see e.g. Proposition 3 in [15].

The short proof of the main theorem is presented in the following section: it is preceded by an outline of Besov spaces and their basic relevant properties, some preliminary material on the structure of the Euler-Korteweg system, followed by he main part of the proof in Sect. 2.3.

\section{Proof of the main theorem}

\subsection{Besov spaces}

Let $\Omega=(0, T) \times \mathbb{T}^{d}$. The Besov space $B_{p}^{\alpha, \infty}(\Omega)$, with $1 \leq p<\infty, 0<\alpha<1$, is the space of functions $w \in L^{p}$ for which the norm

$$
\|w\|_{B_{p}^{\alpha, \infty}(\Omega)}:=\|w\|_{L^{p}(\Omega)}+\sup _{r>0}\left\{r^{-\alpha} \sup _{|\xi| \leq r}\|w(\cdot+\xi)-w\|_{L^{p}(\Omega \cap(\Omega-\xi))}\right\}
$$


is finite, cf. [4]. In fact, we can replace the semi-norm in (2.1) with the following one

$$
\sup _{\xi \in \Omega}\left\{|\xi|^{-\alpha}\|w(\cdot+\xi)-w\|_{L^{p}(\Omega \cap(\Omega-\xi))}\right\} .
$$

Indeed, if $\xi^{*}$ and $r^{*}$ realize the suprema in (2.1) with $\left|\xi^{*}\right|<r^{*}$, then taking $\left|\xi^{*}\right|<r<r^{*}$ would contradict the supremality of $r^{*}$. Therefore neccesarily $\left|\xi^{*}\right|=r^{*}$, thus producing (2.2). We choose to think of the Besov norm in terms of (2.2), as it is more convienient for our purposes.

We observe that if $\alpha \geq \beta$, then there is an inclusion $B_{p}^{\alpha, \infty}(\Omega) \subset B_{p}^{\beta, \infty}(\Omega)$.

Let $\eta \in C_{c}^{\infty}\left(\mathbb{R}^{d+1}\right)$ be a standard mollification kernel and we denote

$$
\eta^{\varepsilon}(x)=\frac{1}{\varepsilon^{d+1}} \eta\left(\frac{x}{\varepsilon}\right), \quad w^{\varepsilon}=\eta^{\varepsilon} * w \quad \text { and } \quad f^{\varepsilon}(w)=f(w) * \eta^{\varepsilon} .
$$

Note that the function $w^{\varepsilon}$ is well-defined on $\Omega^{\varepsilon}=\{x \in \Omega: \operatorname{dist}(x, \partial \Omega)>\varepsilon\}$. The following inequalities will be extensively used in the proof of the main theorem.

Lemma 2.1 For any function $u \in B_{p}^{\alpha, \infty}(\Omega)$ we have

$$
\begin{aligned}
\|u(\cdot+\xi)-u(\cdot)\|_{L^{p}(\Omega \cap(\Omega-\xi))} & \leq|\xi|^{\alpha}\|u\|_{B_{p}^{\alpha, \infty}(\Omega)} \\
\left\|u^{\varepsilon}-u\right\|_{L^{p}(\Omega)} & \leq \varepsilon^{\alpha}\|u\|_{B_{p}^{\alpha, \infty}(\Omega)} \\
\left\|\nabla u^{\varepsilon}\right\|_{L^{p}(\Omega)} & \leq C \varepsilon^{\alpha-1}\|u\|_{B_{p}^{\alpha, \infty}(\Omega)}
\end{aligned}
$$

where $\nabla$ stands here for the space-time gradient.

Proof Inequality (2.3) follows directly from the definition of the norm in the space $B_{p}^{\alpha, \infty}(\Omega)$. To show (2.4) we write

$$
\begin{aligned}
\left|u^{\varepsilon}(x)-u(x)\right| & \leq \int_{\operatorname{supp} \eta^{\varepsilon}} \eta^{\varepsilon}(y)|u(x-y)-u(x)| d y \\
& \leq\left(\int_{\operatorname{supp} \eta^{\varepsilon}} \eta^{\varepsilon}(y)|u(x-y)-u(x)|^{p} d y\right)^{\frac{1}{p}} .
\end{aligned}
$$

Therefore, by virtue of Fubini and (2.3)

$$
\begin{aligned}
\int_{\Omega}\left|u^{\varepsilon}(x)-u(x)\right|^{p} d x & \leq \int_{\operatorname{supp} \eta^{\varepsilon}} \eta^{\varepsilon}(y) \int_{\Omega}|u(x-y)-u(x)|^{p} d x d y \\
& \leq \int_{\operatorname{supp} \eta^{\varepsilon}} \eta^{\varepsilon}(y)|y|^{p \alpha}\|u\|_{B_{p}^{\alpha, \infty}(\Omega)}^{p} d y \leq \varepsilon^{p \alpha}\|u\|_{B_{p}^{\alpha, \infty}(\Omega)}^{p}
\end{aligned}
$$

For the last of the claimed inequalities we consider the convolution $\nabla u^{\varepsilon}=\nabla \eta^{\varepsilon} * u$ as a bounded linear operator $T: L^{p}(\Omega) \rightarrow L^{p}(\Omega)$. Then

$$
\|T u\|_{L^{p}} \leq C \varepsilon^{-1}\|u\|_{L^{p}} .
$$

On the other hand, writing $\nabla u^{\varepsilon}=\eta^{\varepsilon} * \nabla u$, we can think of $T$ as mapping $W^{1, p}(\Omega)$ into $L^{p}(\Omega)$. It then has unit norm.

Therefore, as the Besov space $B_{p}^{\alpha, \infty}$ is an interpolation space of exponent $\alpha$ for $L^{p}$ and $W^{1, p}$ (cf. [4, Corollary 4.13]), $T$ is bounded as an operator $B_{p}^{\alpha, \infty}(\Omega) \rightarrow L^{p}(\Omega)$ with

$$
\|T u\|_{L^{p}} \leq C \varepsilon^{-(1-\alpha)}\|u\|_{B_{p}^{\alpha, \infty} .}
$$


Lemma 2.2 Let $v \in B_{p}^{\alpha, \infty}\left(\Omega, \mathbb{R}^{m}\right)$. Suppose $f: \mathbb{R}^{m} \rightarrow \mathbb{R}$ is a $C^{1}$ function with $\frac{\partial f}{\partial v_{i}} \in L^{\infty}$ for each $i=1, \ldots, m$. Then

$$
\left\|\nabla f\left(v^{\varepsilon}\right)\right\|_{L^{p}} \leq C \varepsilon^{\alpha-1}\|v\|_{B_{p}^{\alpha, \infty}}
$$

Proof Since $\nabla f\left(v^{\varepsilon}\right)=\sum_{i=1}^{m} \frac{\partial f}{\partial v_{i}}\left(v^{\varepsilon}\right) \nabla v_{i}^{\varepsilon}$, we have

$$
\begin{aligned}
\left\|\nabla f\left(v^{\varepsilon}\right)\right\|_{L^{p}} & \leq \sum_{i=1}^{m}\left\|\frac{\partial f}{\partial v_{i}}\left(v^{\varepsilon}\right)\right\|_{L^{\infty}}\left\|\nabla v_{i}^{\varepsilon}\right\|_{L^{p}} \leq \max _{1 \leq i \leq m}\left\|\frac{\partial f}{\partial v_{i}}\right\|_{L^{\infty}} \sum_{i=1}^{m}\left\|\nabla v_{i}^{\varepsilon}\right\|_{L^{p}} \\
& \leq C \varepsilon^{\alpha-1} \sum_{i=1}^{m}\left\|v_{i}\right\|_{B_{p}^{\alpha, \infty}}
\end{aligned}
$$

where the last inequality follows from Lemma 2.1 .

\subsection{Preliminaries}

System (1.1) can be written in conservative form

$$
\begin{aligned}
\partial_{t}(\rho u)+\operatorname{div}(\rho u \otimes u) & =\operatorname{div} \mathbb{S}, \\
\partial_{t} \rho+\operatorname{div}(\rho u) & =0,
\end{aligned}
$$

where $\mathbb{S}$ is the Korteweg stress tensor

$$
\mathbb{S}=\left(-p(\rho)-\frac{\rho \kappa^{\prime}(\rho)+\kappa(\rho)}{2}|\nabla \rho|^{2}+\operatorname{div}(\rho \kappa(\rho) \nabla \rho)\right) \mathbb{I}-\kappa(\rho) \nabla \rho \otimes \nabla \rho
$$

with $\mathbb{I}$ denoting the $d$-dimensional identity matrix and the local pressure defined as

$$
p(\rho)=\rho h^{\prime}(\rho)-h(\rho) .
$$

It is routine to show that a strong solution $(\rho, u)$ of the above system will satisfy the following local balance of total (kinetic and internal) energy

$$
\begin{aligned}
\partial_{t} & \left(\frac{1}{2} \rho|u|^{2}+h(\rho)+\frac{1}{2} \kappa(\rho)|\nabla \rho|^{2}\right) \\
& +\operatorname{div}\left(\rho u\left(\frac{1}{2}|u|^{2}+h^{\prime}(\rho)+\frac{1}{2} \kappa^{\prime}(\rho)|\nabla \rho|^{2}-\operatorname{div}(\kappa(\rho) \nabla \rho)\right)+\kappa(\rho) \nabla \rho \operatorname{div}(\rho u)\right)=0 .
\end{aligned}
$$

Theorem 1.1 gives sufficient conditions for regularity of weak solutions so that they obey the above energy equality in the sense of distributions. To prove the theorem we employ the strategy of [9], which was used in many works in the subject, including [18,22], where variants of the following lemma are an important ingredient.

Lemma 2.3 Let $1 \leq q<\infty$ and suppose $v \in L^{2 q}\left((0, T) \times \mathbb{T}^{d} ; \mathbb{R}^{k}\right)$ and $f \in \mathscr{C}^{2}\left(\mathbb{R}^{k}, \mathbb{R}^{N}\right)$. If

$$
\sup _{i, j}\left\|\frac{\partial^{2} f}{\partial v_{i} \partial v_{j}}\right\|_{L^{\infty}}<\infty
$$


then there exists a constant $C>0$ such that

$$
\left\|f\left(v^{\varepsilon}\right)-f^{\varepsilon}(v)\right\|_{L^{q}} \leq C\left(\left\|v^{\varepsilon}-v\right\|_{L^{2 q}}^{2}+\sup _{(s, y) \in \operatorname{supp} \eta_{\varepsilon}}\|v(\cdot, \cdot)-v(\cdot-s, \cdot-y)\|_{L^{2 q}}^{2}\right) .
$$

Proof We observe that by Taylor's theorem we have

$$
\begin{aligned}
& \left.\mid f\left(v^{\varepsilon}(t, x)\right)-f(v(t, x))\right)-D f(v(t, x))\left(v^{\varepsilon}(t, x)-v(t, x)\right) \mid \\
& \quad \leq C\left|v^{\varepsilon}(t, x)-v(t, x)\right|^{2}
\end{aligned}
$$

where the constant $C$ does not depend on the choice of $x$ and $t$. Similarly

$$
\begin{aligned}
& |f(v(s, y))-f(v(t, x))-D f(v(t, x))(v(s, y)-v(t, x))| \\
& \quad \leq C|v(s, y)-v(t, x)|^{2} .
\end{aligned}
$$

Mollification of the last inequality with respect to $(s, y)$ yields, by virtue of Jensen's inequality

$$
\begin{aligned}
& \mid f^{\varepsilon}(v(t, x))-f\left(v(t, x)-D f(v(t, x))\left(v^{\varepsilon}(t, x)-v(t, x)\right) \mid\right. \\
& \quad \leq C|v(\cdot, \cdot)-v(t, x)|^{2} *_{(s, y)} \eta^{\varepsilon} .
\end{aligned}
$$

Combining (2.9) and (2.11) and using the triangle inequality we deduce the estimate

$$
\begin{aligned}
& \left|f\left(v^{\varepsilon}(t, x)\right)-f^{\varepsilon}(v(t, x))\right| \leq C\left(\left|v^{\varepsilon}(t, x)-v(t, x)\right|^{2}\right. \\
& \left.\quad+|v(\cdot, \cdot)-v(t, x)|^{2} *(s, y) \eta^{\varepsilon}\right) .
\end{aligned}
$$

Finally, we observe that

$$
\begin{aligned}
\int_{(0, T) \times \mathbb{T}^{d}} & || v(\cdot, \cdot)-\left.\left.v(t, x)\right|^{2} *(s, y) \eta^{\varepsilon}\right|^{q} d x d t \\
& \leq \int_{\operatorname{supp} \eta^{\varepsilon}} \eta^{\varepsilon}(s, y) \int_{(0, T) \times \mathbb{T}^{d}}|v(t-s, x-y)-v(t, x)|^{2 q} d x d t d y d s \\
& \leq \sup _{(s, y) \in \operatorname{supp} \eta_{\varepsilon}}\|v(\cdot, \cdot)-v(\cdot-s, \cdot-y)\|_{L^{2 q}}^{2 q} .
\end{aligned}
$$

\subsection{Energy equality}

We begin the proof of the theorem by mollifying the momentum equation in both space and time with kernel and notation as in Sect. 2.1 to obtain

$$
\partial_{t}(\rho u)^{\varepsilon}+\operatorname{div}(\rho u \otimes u)^{\varepsilon}=-\nabla p^{\varepsilon}(\rho)+\operatorname{div} S^{\varepsilon}(\rho, \nabla \rho, \Delta \rho),
$$

where we define

$$
S(\rho, q, r)=\left(\frac{1}{2}\left(\rho \kappa^{\prime}(\rho)+\kappa(\rho)\right)|q|^{2}+\rho \kappa(\rho) r\right) \mathbb{I}-\kappa(\rho) q \otimes q,
$$

and $S^{\varepsilon}(\rho, \nabla \rho, \Delta \rho)=S(\rho, \nabla \rho, \Delta \rho) * \eta^{\varepsilon}$. We note that

$$
S(\rho, \nabla \rho, \Delta \rho)=\left(-\frac{1}{2}\left(\rho \kappa^{\prime}(\rho)+\kappa(\rho)\right)|\nabla \rho|^{2}+\operatorname{div}(\rho \kappa(\rho) \nabla \rho)\right) \mathbb{I}-\kappa(\rho) \nabla \rho \otimes \nabla \rho,
$$


cf. the definition of $\mathbb{S}$.

Equation (2.13) can be rewritten in terms of appropriate commutators to give

$$
\begin{aligned}
& \partial_{t}\left(\rho^{\varepsilon} u^{\varepsilon}\right)+\operatorname{div}\left((\rho u)^{\varepsilon} \otimes u^{\varepsilon}\right)+\nabla p\left(\rho^{\varepsilon}\right)-\operatorname{div}\left(S\left(\rho^{\varepsilon}, \nabla \rho^{\varepsilon}, \Delta \rho^{\varepsilon}\right)\right) \\
& \quad=\partial_{t}\left(\rho^{\varepsilon} u^{\varepsilon}-(\rho u)^{\varepsilon}\right)+\operatorname{div}\left((\rho u)^{\varepsilon} \otimes u^{\varepsilon}-(\rho u \otimes u)^{\varepsilon}\right)+\nabla\left(p\left(\rho^{\varepsilon}\right)-p^{\varepsilon}(\rho)\right) \\
& \quad-\operatorname{div}\left(S\left(\rho^{\varepsilon}, \nabla \rho^{\varepsilon}, \Delta \rho^{\varepsilon}\right)-S^{\varepsilon}(\rho, \nabla \rho, \Delta \rho)\right) .
\end{aligned}
$$

We observe the following identities

$$
\operatorname{div}\left((\rho u)^{\varepsilon} \otimes u^{\varepsilon}\right)=u^{\varepsilon} \operatorname{div}(\rho u)^{\varepsilon}+\left((\rho u)^{\varepsilon} \cdot \nabla\right) u^{\varepsilon}
$$

and

$$
-\rho^{\varepsilon} \nabla\left(\frac{1}{2} \kappa^{\prime}\left(\rho^{\varepsilon}\right)\left|\nabla \rho^{\varepsilon}\right|^{2}-\operatorname{div}\left(\kappa\left(\rho^{\varepsilon}\right) \nabla \rho^{\varepsilon}\right)\right)=\operatorname{div} S\left(\rho^{\varepsilon}, \nabla \rho^{\varepsilon}, \Delta \rho^{\varepsilon}\right) .
$$

Thus the left-hand side of Eq. (2.15) can be written as

$$
\begin{aligned}
\left(\partial_{t} \rho^{\varepsilon}\right) u^{\varepsilon} & +\rho^{\varepsilon} \partial_{t} u^{\varepsilon}+u^{\varepsilon} \operatorname{div}(\rho u)^{\varepsilon}+\left((\rho u)^{\varepsilon} \cdot \nabla\right) u^{\varepsilon} \\
& +\rho^{\varepsilon} \nabla\left(h^{\prime}\left(\rho^{\varepsilon}\right)-\frac{1}{2} \kappa^{\prime}\left(\rho^{\varepsilon}\right)\left|\nabla \rho^{\varepsilon}\right|^{2}-\kappa\left(\rho^{\varepsilon}\right) \Delta \rho^{\varepsilon}\right) .
\end{aligned}
$$

Hence, upon multiplying with $u^{\varepsilon}$, Eq. (2.15) becomes

$$
\begin{aligned}
\rho^{\varepsilon} \partial_{t} & \left(\frac{1}{2}\left|u^{\varepsilon}\right|^{2}\right)+\left((\rho u)^{\varepsilon} \cdot \nabla\right) \frac{1}{2}\left|u^{\varepsilon}\right|^{2} \\
& +\rho^{\varepsilon} u^{\varepsilon} \nabla\left(h^{\prime}\left(\rho^{\varepsilon}\right)-\frac{1}{2} \kappa^{\prime}\left(\rho^{\varepsilon}\right)\left|\nabla \rho^{\varepsilon}\right|^{2}-\kappa\left(\rho^{\varepsilon}\right) \Delta \rho^{\varepsilon}\right) \\
& =r_{1}^{\varepsilon}+r_{2}^{\varepsilon}+r_{3}^{\varepsilon}+r_{4}^{\varepsilon},
\end{aligned}
$$

where

$$
\begin{aligned}
r_{1}^{\varepsilon} & =\partial_{t}\left(\rho^{\varepsilon} u^{\varepsilon}-(\rho u)^{\varepsilon}\right) \cdot u^{\varepsilon}, \\
r_{2}^{\varepsilon} & =\operatorname{div}\left((\rho u)^{\varepsilon} \otimes u^{\varepsilon}-(\rho u \otimes u)^{\varepsilon}\right) \cdot u^{\varepsilon}, \\
r_{3}^{\varepsilon} & =\nabla\left(p\left(\rho^{\varepsilon}\right)-p^{\varepsilon}(\rho)\right) \cdot u^{\varepsilon}, \\
r_{4}^{\varepsilon} & =-\operatorname{div}\left(S\left(\rho^{\varepsilon}, \nabla \rho^{\varepsilon}, \Delta \rho^{\varepsilon}\right)-S^{\varepsilon}(\rho, \nabla \rho, \Delta \rho)\right) \cdot u^{\varepsilon},
\end{aligned}
$$

and we have used the mollified continuity equation

$$
\partial_{t} \rho^{\varepsilon}+\operatorname{div}(\rho u)^{\varepsilon}=0 .
$$

Using (2.17) we can write the first two terms of (2.16) as

$$
\begin{gathered}
\rho^{\varepsilon} \partial_{t}\left(\frac{1}{2}\left|u^{\varepsilon}\right|^{2}\right)+\left((\rho u)^{\varepsilon} \cdot \nabla\right) \frac{1}{2}\left|u^{\varepsilon}\right|^{2}+\left(\partial_{t} \rho^{\varepsilon}+\operatorname{div}(\rho u)^{\varepsilon}\right) \frac{1}{2}\left|u^{\varepsilon}\right|^{2} \\
=\partial_{t}\left(\frac{1}{2} \rho^{\varepsilon}\left|u^{\varepsilon}\right|^{2}\right)+\operatorname{div}\left((\rho u)^{\varepsilon} \frac{1}{2}\left|u^{\varepsilon}\right|^{2}\right) .
\end{gathered}
$$

Combining Eqs. (2.16) and (2.18) we obtain

$$
\begin{aligned}
\partial_{t}( & \left.\frac{1}{2} \rho^{\varepsilon}\left|u^{\varepsilon}\right|^{2}\right)+\operatorname{div}\left((\rho u)^{\varepsilon} \frac{1}{2}\left|u^{\varepsilon}\right|^{2}\right) \\
& +\rho^{\varepsilon} u^{\varepsilon} \nabla\left(h^{\prime}\left(\rho^{\varepsilon}\right)-\frac{1}{2} \kappa^{\prime}\left(\rho^{\varepsilon}\right)\left|\nabla \rho^{\varepsilon}\right|^{2}-\kappa\left(\rho^{\varepsilon}\right) \Delta \rho^{\varepsilon}\right) \\
& =r_{1}^{\varepsilon}+r_{2}^{\varepsilon}+r_{3}^{\varepsilon}+r_{4}^{\varepsilon} .
\end{aligned}
$$


We now rewrite the mollified continuity Eq. (2.17) in the form

$$
\partial_{t} \rho^{\varepsilon}+\operatorname{div}\left(\rho^{\varepsilon} u^{\varepsilon}\right)=\operatorname{div}\left(\rho^{\varepsilon} u^{\varepsilon}-(\rho u)^{\varepsilon}\right) .
$$

After multiplying this equation with

$$
h^{\prime}\left(\rho^{\varepsilon}\right)-\frac{1}{2} \kappa^{\prime}\left(\rho^{\varepsilon}\right)\left|\nabla \rho^{\varepsilon}\right|^{2}-\kappa\left(\rho^{\varepsilon}\right) \Delta \rho^{\varepsilon}
$$

and rearranging, we obtain

$$
\begin{aligned}
& \partial_{t}\left(h\left(\rho^{\varepsilon}\right)+\frac{1}{2} \kappa\left(\rho^{\varepsilon}\right)\left|\nabla \rho^{\varepsilon}\right|^{2}\right)-\operatorname{div}\left(\kappa\left(\rho^{\varepsilon}\right) \nabla \rho^{\varepsilon} \partial_{t} \rho^{\varepsilon}\right) \\
& \quad+\operatorname{div}\left(\rho^{\varepsilon} u^{\varepsilon}\right)\left(h^{\prime}\left(\rho^{\varepsilon}\right)-\frac{1}{2} \kappa^{\prime}\left(\rho^{\varepsilon}\right)\left|\nabla \rho^{\varepsilon}\right|^{2}-\kappa\left(\rho^{\varepsilon}\right) \Delta \rho^{\varepsilon}\right) \\
& =r_{5}^{\varepsilon}+r_{6}^{\varepsilon}+r_{7}^{\varepsilon},
\end{aligned}
$$

where

$$
\begin{aligned}
& r_{5}^{\varepsilon}=\operatorname{div}\left(\rho^{\varepsilon} u^{\varepsilon}-(\rho u)^{\varepsilon}\right) h^{\prime}\left(\rho^{\varepsilon}\right), \\
& r_{6}^{\varepsilon}=-\operatorname{div}\left(\rho^{\varepsilon} u^{\varepsilon}-(\rho u)^{\varepsilon}\right) \frac{1}{2} \kappa^{\prime}\left(\rho^{\varepsilon}\right)\left|\nabla \rho^{\varepsilon}\right|^{2}, \\
& r_{7}^{\varepsilon}=-\operatorname{div}\left(\rho^{\varepsilon} u^{\varepsilon}-(\rho u)^{\varepsilon}\right) \kappa\left(\rho^{\varepsilon}\right) \Delta \rho^{\varepsilon} .
\end{aligned}
$$

Combining Eqs. (2.19) and (2.20) we obtain

$$
\begin{aligned}
\partial_{t}( & \left.\frac{1}{2} \rho^{\varepsilon}\left|u^{\varepsilon}\right|^{2}+h\left(\rho^{\varepsilon}\right)+\frac{1}{2} \kappa\left(\rho^{\varepsilon}\right)\left|\nabla \rho^{\varepsilon}\right|^{2}\right)+\operatorname{div}\left((\rho u)^{\varepsilon} \frac{1}{2}\left|u^{\varepsilon}\right|^{2}\right) \\
& +\operatorname{div}\left(\rho^{\varepsilon} u^{\varepsilon}\left(h^{\prime}\left(\rho^{\varepsilon}\right)-\frac{1}{2} \kappa^{\prime}\left(\rho^{\varepsilon}\right)\left|\nabla \rho^{\varepsilon}\right|^{2}-\kappa\left(\rho^{\varepsilon}\right) \Delta \rho^{\varepsilon}\right)+\kappa\left(\rho^{\varepsilon}\right) \nabla \rho^{\varepsilon} \operatorname{div}\left(\rho^{\varepsilon} u^{\varepsilon}\right)\right) \\
& =r_{1}^{\varepsilon}+r_{2}^{\varepsilon}+r_{3}^{\varepsilon}+r_{4}^{\varepsilon}+r_{5}^{\varepsilon}+r_{6}^{\varepsilon}+r_{7}^{\varepsilon} .
\end{aligned}
$$

It follows that to prove the theorem it is sufficient to show that each commutator error term converges to zero in the distributional sense on $(0, T) \times \mathbb{T}^{d}$ as $\varepsilon \rightarrow 0$.

\subsection{Commutator estimates}

Let $\varphi \in \mathscr{C}_{c}^{1}\left((0, T) \times \mathbb{T}^{d}\right)$ and take $\varepsilon>0$ small enough so that $\operatorname{supp} \varphi \subset(\varepsilon, T-\varepsilon) \times \mathbb{T}^{d}$. We will show that for each $1 \leq i \leq 7$ we have

$$
R_{i}^{\varepsilon}:=\int_{0}^{T} \int_{\mathbb{T}^{d}} r_{i}^{\varepsilon} \varphi d x d t \stackrel{\varepsilon \rightarrow 0^{+}}{\longrightarrow} 0 .
$$

The terms $R_{1}^{\varepsilon}$ and $R_{2}^{\varepsilon}$ are dealt with in the same way as in [18]. We recall these estimates for the reader's convenience. For $R_{1}^{\varepsilon}$ we observe that

$$
\begin{aligned}
\rho^{\varepsilon} u^{\varepsilon}-(\rho u)^{\varepsilon}= & \left(\rho^{\varepsilon}-\rho\right)\left(u^{\varepsilon}-u\right) \\
& -\int_{-\varepsilon}^{\varepsilon} \int_{\mathbb{T}^{d}} \eta^{\varepsilon}(\tau, \xi)(\rho(t-\tau, x-\xi) \\
& -\rho(t, x))(u(t-\tau, x-\xi)-u(t, x)) d \xi d \tau .
\end{aligned}
$$


The first part of $R_{1}^{\varepsilon}$ therefore can be estimated by virtue of an integration by parts, Hölder inequality and estimates (2.4) and (2.5) as

$$
\begin{aligned}
& \left|\int_{0}^{T} \int_{\mathbb{T}^{d}} \varphi \partial_{t}\left(\left(\rho^{\varepsilon}-\rho\right)\left(u^{\varepsilon}-u\right)\right) \cdot u^{\varepsilon} d x d t\right| \\
& \leq \int_{0}^{T} \int_{\mathbb{T}^{d}}\left|\left(\rho^{\varepsilon}-\rho\right)\left(u^{\varepsilon}-u\right)\right|\left(\left|\partial_{t} \varphi u^{\varepsilon}\right|+\left|\varphi \partial_{t} u^{\varepsilon}\right|\right) d x d t \\
& \leq\|\varphi\|_{\mathscr{C}^{1}}\left\|\rho^{\varepsilon}-\rho\right\|_{L^{3}}\left\|u^{\varepsilon}-u\right\|_{L^{3}}\left\|u^{\varepsilon}\right\|_{L^{3}}+\|\varphi\|_{\mathscr{C}^{0}}\left\|\rho^{\varepsilon}-\rho\right\|_{L^{3}}\left\|u^{\varepsilon}-u\right\|_{L^{3}}\left\|\partial_{t} u^{\varepsilon}\right\|_{L^{3}} \\
& \leq C \varepsilon^{\beta} \varepsilon^{\alpha}\|\rho\|_{B_{3}^{\beta, \infty}}\|u\|_{B_{3}^{\alpha, \infty}}^{2}+C \varepsilon^{\beta} \varepsilon^{\alpha} \varepsilon^{\alpha-1}\|\rho\|_{B_{3}^{\beta, \infty}}\|u\|_{B_{3}^{\alpha, \infty}}^{2} \text {. }
\end{aligned}
$$

Note that since we assume Besov regularity of $u$ also in time, in the above we can estimate the $L^{3}$-norm of $\partial_{t} u^{\varepsilon}$ according to (2.5).

For the second part of $R_{1}^{\varepsilon}$ according to (2.22), we estimate (using integration by parts, Fubini (2.3) and (2.5))

$$
\begin{aligned}
& \mid \int_{0}^{T} \int_{\mathbb{T}^{d}} \varphi \partial_{t}\left(\int_{-\varepsilon}^{\varepsilon} \int_{\mathbb{T}^{d}} \eta^{\varepsilon}(\tau, \xi)(\rho(t-\tau, x-\xi)\right. \\
& -\rho(t, x))(u(t-\tau, x-\xi)-u(t, x)) d \xi d \tau) \cdot u^{\varepsilon} d x d t \mid \\
& \leq C\|\varphi\|_{\mathscr{C}^{1}} \varepsilon^{\beta} \varepsilon^{\alpha}\|\rho\|_{B_{3}^{\beta, \infty}}\|u\|_{B_{3}^{\alpha, \infty}}^{2}+C\|\varphi\|_{\mathscr{C}^{0}} \varepsilon^{\beta} \varepsilon^{\alpha} \varepsilon^{\alpha-1}\|\rho\|_{B_{3}^{\beta, \infty}}\|u\|_{B_{3}^{\alpha, \infty}}^{2} .
\end{aligned}
$$

A similar estimation can be carried out for $R_{2}^{\varepsilon}$. We write

$$
\begin{aligned}
& (\rho u)^{\varepsilon} \otimes u^{\varepsilon}-(\rho u \otimes u)^{\varepsilon}=\left((\rho u)^{\varepsilon}-\rho u\right) \otimes\left(u^{\varepsilon}-u\right) \\
& -\int_{-\varepsilon}^{\varepsilon} \int_{\mathbb{T}^{d}} \eta^{\varepsilon}(\tau, \xi)(\rho u(t-\tau, x-\xi)-\rho u(t, x)) \\
& \otimes(u(t-\tau, x-\xi)-u(t, x)) d \xi d \tau .
\end{aligned}
$$

To use the above decomposition to estimate $R_{2}^{\varepsilon}$ we require that the product $\rho u$ belongs to the space $B_{3}^{\beta, \infty}\left((0, T) \times \mathbb{T}^{d}\right)$. To provide this regularity we needed to assume not only that $\rho$ and $u$ are in appropriate Besov spaces, but also belong to $L^{\infty}$. Indeed, observe that since $\alpha \geq \beta$, we have $u \in B_{3}^{\beta, \infty}$ and

$$
\begin{aligned}
\frac{\|(\rho u)(\cdot+\xi)-\rho u\|_{L^{3}}}{|\xi|^{\beta}} & \leq \frac{\|\rho(u(\cdot+\xi)-u)\|_{L^{3}}}{|\xi|^{\beta}}+\frac{\|(\rho(\cdot+\xi)-\rho) u(\cdot+\xi)\|_{L^{3}}}{|\xi|^{\beta}} \\
& \leq\|\rho\|_{L^{\infty}}\|u\|_{B_{3}^{\beta, \infty}}+\|u\|_{L^{\infty}}\|\rho\|_{B_{3}^{\beta, \infty}}
\end{aligned}
$$

Thus the first part of $R_{2}^{\varepsilon}$ can be estimated as

$$
\begin{aligned}
& \left|\int_{0}^{T} \int_{\mathbb{T}^{d}} \operatorname{div}\left(\left((\rho u)^{\varepsilon}-\rho u\right) \otimes\left(u^{\varepsilon}-u\right)\right) \cdot \varphi u^{\varepsilon} d x d t\right| \\
& \quad \leq\|\varphi\|_{C^{1}}\left\|(\rho u)^{\varepsilon}-\rho u\right\|_{L^{3}}\left\|u^{\varepsilon}-u\right\|_{L^{3}}\left\|u^{\varepsilon}\right\|_{L^{3}} \\
& \quad+\|\varphi\|_{C^{0}}\left\|(\rho u)^{\varepsilon}-\rho u\right\|_{L^{3}}\left\|u^{\varepsilon}-u\right\|_{L^{3}}\left\|\nabla u^{\varepsilon}\right\|_{L^{3}} \\
& \quad \leq C \varepsilon^{\beta} \varepsilon^{\alpha}\|\rho\|_{B_{3}^{\beta, \infty}}\|u\|_{B_{3}^{\alpha, \infty}}^{2}+C \varepsilon^{\beta} \varepsilon^{\alpha} \varepsilon^{\alpha-1}\|\rho u\|_{B_{3}^{\beta, \infty}}\|u\|_{B_{3}^{\alpha, \infty}}^{2} .
\end{aligned}
$$


Likewise, for the second part of $R_{2}^{\varepsilon}$ we get

$$
\begin{aligned}
& \mid \int_{0}^{T} \int_{\mathbb{T}^{d}} \operatorname{div}\left\{\int_{-\varepsilon}^{\varepsilon} \int_{\mathbb{T}^{d}} \eta^{\varepsilon}(\tau, \xi)(\rho u(t-\tau, x-\xi)-\rho u(t, x))\right. \\
& \otimes(u(t-\tau, x-\xi)-u(t, x)) d \xi d \tau\} \cdot \varphi u^{\varepsilon} d x d t \\
& \leq C\|\varphi\|_{\mathscr{C}^{0}} \varepsilon^{\beta} \varepsilon^{\alpha} \varepsilon^{\alpha-1}\|\rho u\|_{B_{3}^{\beta, \infty}}\|u\|_{B_{3}^{\alpha, \infty}}^{2}+C\|\varphi\|_{\mathscr{C}^{1}} \varepsilon^{\beta} \varepsilon^{\alpha}\|\rho\|_{B_{3}^{\beta, \infty}}\|u\|_{B_{3}^{\alpha, \infty}}^{2} .
\end{aligned}
$$

These estimates show that $R_{1}^{\varepsilon}$ and $R_{2}^{\varepsilon}$ vanish as $\varepsilon \rightarrow 0$.

To estimate terms $R_{3}^{\varepsilon}$ and $R_{4}^{\varepsilon}$ we integrate by parts and apply Lemma 2.3 to get the following

$$
\begin{aligned}
\left|R_{3}^{\varepsilon}\right| \leq & \|\varphi\|_{\mathscr{C}^{1}} \int_{0}^{T} \int_{\mathbb{T}^{d}}\left|p\left(\rho^{\varepsilon}\right)-p^{\varepsilon}(\rho)\left\|u^{\varepsilon}\left|d x d t+\|\varphi\|_{\mathscr{C}^{0}} \int_{0}^{T} \int_{\mathbb{T}^{d}}\right| p\left(\rho^{\varepsilon}\right)\right.\right. \\
& -p^{\varepsilon}(\rho) \| \nabla u^{\varepsilon} \mid d x d t \\
\leq & C\left\|p\left(\rho^{\varepsilon}\right)-p^{\varepsilon}(\rho)\right\|_{L^{3 / 2}}\left(\left\|u^{\varepsilon}\right\|_{L^{3}}+\left\|\nabla u^{\varepsilon}\right\|_{L^{3}}\right) \\
\leq & C\left(\left\|\rho^{\varepsilon}-\rho\right\|_{L^{3}}^{2}+\sup _{y \in \operatorname{supp} \eta^{\varepsilon}}\|\rho(\cdot)-\rho(\cdot-y)\|_{L^{3}}^{2}\right)\left(1+\varepsilon^{\alpha-1}\right)\|u\|_{B_{3}^{\alpha, \infty}} \\
\leq & C\left(\varepsilon^{2 \beta}\|\rho\|_{B_{3}^{\beta, \infty}}^{2}+\sup _{|y| \leq \varepsilon}|y|^{2 \beta}\|\rho\|_{B_{3}^{\beta, \infty}}^{2}\right)\left(1+\varepsilon^{\alpha-1}\right)\|u\|_{B_{3}^{\alpha, \infty}} \\
\leq & C\left(\varepsilon^{2 \beta}+\varepsilon^{2 \beta+\alpha-1}\right)\|u\|_{B_{3}^{\alpha, \infty}\|\rho\|_{B_{3}^{\beta, \infty}}^{2}}
\end{aligned}
$$

and similarly

$$
\begin{aligned}
\left|R_{4}^{\varepsilon}\right| & \leq C\left\|S\left(\rho^{\varepsilon}, \nabla \rho^{\varepsilon}, \Delta \rho^{\varepsilon}\right)-S^{\varepsilon}(\rho, \nabla \rho, \Delta \rho)\right\|_{L^{3 / 2}}\left(\left\|u^{\varepsilon}\right\|_{L^{3}}+\left\|\nabla u^{\varepsilon}\right\|_{L^{3}}\right) \\
& \leq C\left(\varepsilon^{2 \beta}+\varepsilon^{2 \beta+\alpha-1}\right)\|u\|_{B_{3}^{\alpha, \infty}}\left(\|\rho\|_{B_{3}^{\beta, \infty}}^{2}+\|\nabla \rho\|_{B_{3}^{\beta, \infty}}^{2}+\|\Delta \rho\|_{B_{3}^{\beta, \infty}}^{2}\right) .
\end{aligned}
$$

It now remains to estimate the last three commutator errors $R_{5}^{\varepsilon}, R_{6}^{\varepsilon}$ and $R_{7}^{\varepsilon}$. To this end we employ Lemma 2.2 with function $f$ being $h^{\prime}(\rho), \kappa^{\prime}(\rho)|\nabla \rho|^{2}$, and $\kappa(\rho) \Delta \rho$, respectively. We observe that by assumptions (1.2) and (1.4) these functions belong to $L^{\infty}$. Using again equality (2.22) we can estimate as follows.

$$
\begin{aligned}
\left|R_{5}^{\varepsilon}\right| \leq & \int_{0}^{T} \int_{\mathbb{T}^{d}}\left|\left(\rho^{\varepsilon}-\rho\right)\left(u^{\varepsilon}-u\right)\right|\left(\left|h^{\prime}\left(\rho^{\varepsilon}\right) \nabla \varphi\right|+\left|\varphi \nabla h^{\prime}\left(\rho^{\varepsilon}\right)\right|\right) d x d t \\
\leq & \|\varphi\|_{\mathscr{C}^{1}}\left\|\rho^{\varepsilon}-\rho\right\|_{L^{3}}\left\|u^{\varepsilon}-u\right\|_{L^{3}}\left\|h^{\prime}\left(\rho^{\varepsilon}\right)\right\|_{L^{3}} \\
& +\|\varphi\|_{\mathscr{C}^{0}}\left\|\rho^{\varepsilon}-\rho\right\|_{L^{3}}\left\|u^{\varepsilon}-u\right\|_{L^{3}}\left\|\nabla h^{\prime}\left(\rho^{\varepsilon}\right)\right\|_{L^{3}} \\
\leq & C \varepsilon^{\beta} \varepsilon^{\alpha}\|\rho\|_{B_{3}^{\beta, \infty}}\|u\|_{B_{3}^{\alpha, \infty}}+C \varepsilon^{\beta} \varepsilon^{\alpha} \varepsilon^{\beta-1}\|\rho\|_{B_{3}^{\beta, \infty}}^{2}\|u\|_{B_{3}^{\alpha, \infty},} \\
\left|R_{6}^{\varepsilon}\right| \leq & C\left(\left\|\kappa^{\prime}\left(\rho^{\varepsilon}\right)\left|\nabla \rho^{\varepsilon}\right|^{2}\right\|_{L^{3}}+\left\|\nabla\left(\kappa^{\prime}\left(\rho^{\varepsilon}\right)\left|\nabla \rho^{\varepsilon}\right|^{2}\right)\right\|_{L^{3}}\right)\left\|\rho^{\varepsilon}-\rho\right\|_{L^{3}}\left\|u^{\varepsilon}-u\right\|_{L^{3}} \\
\leq & C \varepsilon^{\beta} \varepsilon^{\alpha}\|\rho\|_{B_{3}^{\beta, \infty}}^{\beta, u}\left\|_{B_{3}^{\alpha, \infty}}+C \varepsilon^{\beta} \varepsilon^{\alpha} \varepsilon^{\beta-1}\right\| \rho\left\|_{B_{3}^{\beta, \infty}}^{2}\right\| u \|_{B_{3}^{\alpha, \infty},}
\end{aligned}
$$

and

$$
\begin{aligned}
& \left|R_{7}^{\varepsilon}\right| \leq C\left(\left\|\kappa\left(\rho^{\varepsilon}\right) \Delta \rho^{\varepsilon}\right\|_{L^{3}}+\left\|\nabla\left(\kappa\left(\rho^{\varepsilon}\right) \Delta \rho^{\varepsilon}\right)\right\|_{L^{3}}\right)\left\|\rho^{\varepsilon}-\rho\right\|_{L^{3}}\left\|u^{\varepsilon}-u\right\|_{L^{3}} \\
& \leq C \varepsilon^{\beta} \varepsilon^{\alpha}\|\rho\|_{B_{3}^{\beta, \infty}}\|u\|_{B_{3}^{\alpha, \infty}}+C \varepsilon^{\beta} \varepsilon^{\alpha} \varepsilon^{\beta-1}\|\rho\|_{B_{3}^{\beta, \infty}}^{2}\|u\|_{B_{3}^{\alpha, \infty}} .
\end{aligned}
$$

For brevity the above calculations include only the first term coming from (2.22), with the second term easily seen to produce estimates of the same order.

Thus the proof of the theorem is complete. 
Acknowledgements This work was partially supported by the Simons - Foundation Grant 346300 and the Polish Government MNiSW 2015-2019 matching fund; AET thanks the Institute of Mathematics of the Polish Academy of Sciences, Warsaw, for their hospitality during his stay as a Simons Visiting Professor, while P.G. and A.S-G thank KAUST for its hospitality during their stay. P.G. and A.S-G. received support from the National Science Centre (Poland), 2015/18/M/ST1/00075. T.D acknowledges the support of the National Science Centre (Poland), 2012/05/E/ST1/02218.

Open Access This article is distributed under the terms of the Creative Commons Attribution 4.0 International License (http://creativecommons.org/licenses/by/4.0/), which permits unrestricted use, distribution, and reproduction in any medium, provided you give appropriate credit to the original author(s) and the source, provide a link to the Creative Commons license, and indicate if changes were made.

\section{References}

1. Antonelli, P., Marcati, P.: On the finite energy weak solutions to a system in quantum fluid dynamics. Commun. Math. Phys. 287(2), 657-686 (2009)

2. Antonelli, P., Marcati, P.: The quantum hydrodynamics system in two space dimensions. Arch. Ration. Mech. Anal. 203(2), 499-527 (2012)

3. Bardos, C., Titi, E.: Onsager's conjecture for the incompressible Euler equations in bounded domains. Arch. Ration. Mech. Anal. 228, 197 (2017). https://doi.org/10.1007/s00205-017-1189-X

4. Bennett, C., Sharpley, R.: Interpolation of Operators. Pure and Applied Mathematics, vol. 129. Academic Press Inc., Boston (1988)

5. Buckmaster, T., De Lellis, C., Székelyhidi Jr., L., Vicol, V.: Onsager's conjecture for admissible weak solutions. Commun. Pure Appl. Math. (2018). https://doi.org/10.1002/cpa.21781

6. Benzoni-Gavage, S., Danchin, R., Descombes, S.: On the well-posedness for the Euler-Korteweg model in several space dimensions. Indiana Univ. Math. J. 56, 1499-1579 (2007)

7. Benzoni-Gavage, S., Danchin, R., Descombes, S., Jamet, D.: Structure of Korteweg models and stability of diffuse interfaces. Interfaces Free Bound. 7(4), 371-414 (2005)

8. Caflisch, R.E., Klapper, I., Steele, G.: Remarks on singularities, dimension and energy dissipation for ideal hydrodynamics and MHD. Commun. Math. Phys. 184(2), 443-455 (1997)

9. Constantin, P., W, E., Titi, E.S.: Onsager's conjecture on the energy conservation for solutions of Euler's equation. Commun. Math. Phys. 165(1), 207-209 (1994)

10. De Lellis, C., Székelyhidi Jr., L.: The Euler equations as a differential inclusion. Ann. Math. (2) 170(3), 1417-1436 (2009)

11. De Lellis, C., Székelyhidi Jr., L.: On admissibility criteria for weak solutions of the Euler equations. Arch. Ration. Mech. Anal. 195, 225-260 (2010)

12. Dębiec, T., Gwiazda, P., Świerczewska-Gwiazda, A.: A tribute to energy conservation for weak solutions. arXiv:1707.09794 (2017)

13. Donatelli, D., Feireisl, E., Marcati, P.: Well/ill posedness for the Euler-Korteweg-Poisson system and related problems. Commun. Partial Differ. Equ. 40(7), 1314-1335 (2015)

14. Drivas, T.D., Eyink, G.L.: An Onsager singularity theorem for turbulent solutions of compressible Euler equations. Commun. Math. Phys. 359, 733 (2017)

15. Duchon, J., Robert, R.: Inertial energy dissipation for weak solutions of incompressible Euler and NavierStokes equations. Nonlinearity 13(1), 249-255 (2000)

16. Dunn, J.E., Serrin, J.: On the thermomechanics of interstitial working. Arch. Ration. Mech. Anal. 88, 95-133 (1985)

17. Eyink, G.L.: Energy dissipation without viscosity in ideal hydrodynamics. I. Fourier analysis and local energy transfer. Physica D 78(3-4), 222-240 (1994)

18. Feireisl, E., Gwiazda, P., Świerczewska-Gwiazda, A., Wiedemann, E.: Regularity and energy conservation for the compressible Euler equations. Arch. Ration. Mech. Anal. 223(3), 1-21 (2017)

19. Gasser, I., Markowich, P.: Quantum hydrodynamics, Wigner transforms and the classical limit. Asymptot. Anal. 14, 97-116 (1997)

20. Giesselmann, J., Tzavaras, A.: Stability properties of the Euler-Korteweg system with nonmonotone pressures. Appl. Anal. 96(9), 1528-1546 (2017)

21. Giesselman, J., Lattanzio, C., Tzavaras, A.: Relative energy for the Korteweg theory and related hamiltonian flows in gas dynamics. Arch. Ration. Mech. Anal. 223(3), 1427-1484 (2017)

22. Gwiazda, P., Michálek, M., Świerczewska-Gwiazda, A.: A note on weak solutions of conservation laws and energy/entropy conservation. Arch. Ration. Mech. Anal. 229(3), 1223-1238 (2018) 
23. Isett, P.: A proof of Onsager's conjecture. Ann. Math. (2018, to appear)

24. Isett, P.: On the endpoint regularity in Onsager's conjecture. arXiv:1706.0154 (2017)

25. Kang, E., Lee, J.: Remarks on the magnetic helicity and energy conservation for ideal magnetohydrodynamics. Nonlinearity 20(11), 2681-2689 (2007)

26. Leslie, T.M., Shvydkoy, R.: The energy balance relation for weak solutions of the density-dependent Navier-Stokes equations. J. Differ. Equ. 261(6), 3719-3733 (2016)

27. Onsager, L.: Statistical hydrodynamics. Nuovo Cimento (9) 6, 279-287 (1949). (Convegno Internazionale di Meccanica Statistica)

28. Scheffer, V.: An inviscid flow with compact support in space-time. J. Geom. Anal. 3(4), 343-401 (1993)

29. Shnirelman, A.: Weak solutions with decreasing energy of incompressible Euler equations. Commun. Math. Phys. 210(3), 541-603 (2000)

30. Shvydkoy, R.: On the energy of inviscid singular flows. J. Math. Anal. Appl. 349, 583-595 (2009)

31. Yu, C.: Energy conservation for the weak solutions of the compressible Navier-Stokes equations. Arch. Ration. Mech. Anal. 225(2), 1073-1087 (2017) 